\title{
Metastatic osteosarcoma challenged by regorafenib
}

Osteosarcomas belong to a large family of tumour entities of mesenchymal origin which exhibit heterogeneous histological, genetic and molecular features. Their pathogenesis can be explained by initial TP53 and/or RB1 somatic alterations leading to chromosomic instability, followed by secondary oncogenic events and the development of a polyclonal disease associated with the metastatic process. This genetic complexity has been illustrated by recent series of patients in which the authors have identified a considerable number of point mutations and deletions in an impressive number of genes. ${ }^{1}$ It has also stimulated the development of numerous therapeutic strategies targeting tumour cells and/or their microenvironment. ${ }^{2}$ The fact that osteosarcomas are both rare forms of cancer and highly heterogeneous explains why the patient survival rate has not improved in the last four decades, especially for metastatic and unresectable osteosarcomas. Regardless of whether or not the drugs used in the first line of chemotherapy (in neoadjuvant and/or adjuvant chemotherapy for 6 to 12 months) have been standardized (relatively speaking) and include doxorubicin, cisplatin, methotrexate and ifosfamide, there is no consensus on either the optimum combination or the therapeutic options for recurrent metastatic patients. ${ }^{3}$

Protein kinases are part of a large family of key enzymes that catalyse the transfer of a phosphate group from ATP to a hydroxyl group of serine or threonine. More than 50 protein kinases are receptors (receptor tyrosine kinase: RTK) and are classified into 6 classes of protein, depending on their biochemical structure. ${ }^{4}$ All RTK are characterized by a single trans-membrane domain and a glycosylated $\mathrm{N}$-terminal extracellular domain involved in both the dimerization mechanism of the receptors, and in the ligand recognition that triggers downstream signalling pathways, including MAPK, PI3K, Src, PLC $\gamma$, JAK / STAT. On the basis of their diversity, and their wide-ranging expression in normal cells, cancer cells and the signalling cascades they trigger, RTK are strongly implicated in multiple pathways of cancer development. As a result, numerous RTK inhibitors have been developed by pharmaceutical companies. Imatinib mesylate $\left(\right.$ Gleevec $\left.^{\circledR}\right)$ was the first to demonstrate spectacular clinical benefits in chronic myeloid leukaemia (CML) patients and in gastro-intestinal stromal tumours (GIST), and it has become the standard treatment in patients with a high risk of GIST recurrence following tumour resection. Regorafenib belongs to the long list of multikinase 
inhibitors with non-specific activities targeting VGFR1/2/3, RET, Raf, PDGFR and KIT. Interestingly, in a phase I clinical trial regorafenib produced an objective response in one of three patients with a second-line advanced osteosarcoma. ${ }^{5}$

In The Lancet Oncology, Florence Duffaud et coll. ${ }^{6}$ report the results of regorafenib in patients with advanced osteosarcoma, in a non-comparative, randomized, double-blind placebo-controlled phase 2 clinical trial. The authors observed 16-week progression-free survival (95\%, CI 8.0-27.3) in the tyrosine kinase inhibitor arm and 4 weeks (95\%, CI 3.05.7) in the placebo group. This difference was maintained over time at 12 and 24 weeks, with a non-progression rate of $35 \%$ for the patients treated with regorafenib and $0 \%$ for those on placebo. Although the present study was statistically non-comparative, it highlights the potential therapeutic value of regorafenib in cases of recurrent and unresectable osteosarcoma for which there are currently only limited therapeutic options and a pressing medical need.

So what is next? The next step will be to identify the main cellular and molecular targets in the patient population of interest. The questions are whether the therapeutic benefits of regorafenib are linked to the targeting of a single or multiple RTK, and on which cells? Are the effects of regorafenib related to direct activity on cancer cells and/or on cells present in their microenvironment? Identifying the target(s) of regorafenib is essential for moving towards better stratification of the patients enrolled in future clinical trials. In addition, approximately $30 \%$ of RTKs are overexpressed or mutated in human cancers and can be associated with drug resistance. ${ }^{7-9}$ In this context, an adapted investigational strategy will be needed to obtain real and precise mapping of activated/mutated/expressed tyrosine kinase proteins.. Previous preclinical and clinical studies have underlined the risk of drug resistance and relapse related to protein point mutations and/or the establishment of molecular feedback loops after administration of RTK inhibitors, as observed in GIST. ${ }^{10}$ A sequential therapeutic approach combining RTK inhibitors, chemotherapy and targeted therapies (e.g. check point inhibitors) may be an alternative for preventing/limiting acquired drug resistance. Pertinent screening of recurrent unresectable osteosarcoma patients on the basis of their RTK profiling could help prevent the development of drug resistance, and thus define the therapeutic alternatives. By increasing the homogeneity of the population studied, this type of screening will also improve the chances of success of large phase 3 clinical trials which, as recommended by the authors, should be set up at the European and international levels. 
Dominique Heymann

- Institut de Cancérologie de l'Ouest, INSERM, CRCINA, UMR 1232, Université de Nantes, Université d'Angers, Blvd Jacques Monod, 44805 Saint-Herblain, France.

- University of Sheffield, Department of Oncology and Metabolism, INSERM, European Associated Laboratory "Sarcoma Research Unit", Medical School, Beech Hill Road, S10 2RX, Sheffield, UK.

dominique.heymann@univ-nantes.fr

I declare no conflict of interest.

1 Bousquet M, Noirot C, Accadbled F, et al. Whole-exome sequencing in osteosarcoma reveals important heterogeneity of genetic alterations. Ann Oncol 2016; 27:738-44.

2 Heymann MF, Brown HK, Heymann D. Drugs in early clinical development for the treatment of osteosarcoma. Expert Opin Investig Drugs 2016; 25:1265-80.

3 Casali PG, Bielack S, Abecassis N, et al. Bone sarcomas: ESMO-PaedCanEURACAN Clinical Practice Guidelines for diagnosis, treatment and follow-up. Ann Oncol 2018; 29: iv79-iv95.

4 Ségaliny AI, Tellez-Gabriel M, Heymann MF, Heymann D. Receptor tyrosine kinase : characterisation, mechanism of action and therapeutic interests for bone cancers. $J$ Bone Oncol 2015; 4:1-12.

5 Mross K, Frost A, Steinbild S, et al. A Phase I dose-escalation study of regorafenib (BAY 73-4506), an inhibitor of oncogenic, angiogenic, and stromal kinases, in patients with advanced solid tumors. Clin Cancer Res 2012,18: 2658-67.

6 Duffaud F, Mir O, Boudu-Rouquette P, Piperno-Neumann S, et al. Efficacy and safety of regorafenib in adult patients with metastatic osteosarcoma: results of a non comparative, randomised, double-blind, placebo controlled, multicentre phase II study. Lancet Oncol $2018 ; \boldsymbol{X} \boldsymbol{X} \boldsymbol{X}$.

7 Kaitsiotou H, Keul M, Hardick J, et al. Inhibitors to overcome secondary mutations in the stem cell factor receptor KIT. J Med Chem 2017;60:8801-15.

8 Lynch TJ, Bell DW, Sordella R, et al. Activating mutations in the epidermal growth factor receptor underlying responsiveness of non-small-cell lung cancer to gefitinib. $N$ Engl $J$ Med 2004;350:2129-39.

9 Bose R, Kavuri SM, Searleman AC, et al. Activating HER2 mutations in HER2 gene amplification negative breast cancer. Cancer Discov 2013;3:224-37.

10 Hemming ML, Heinrich MC, Bauer S, George S. Translational insights into 
gastrointestinal stromal tumor and current clinical advances. Ann Oncol 2018 ; in press. 